\title{
(S)-1,2-Propanediol as the Simplest Acyclic Template for the Asymmetric Induction in Cyclocopolymerization
}

\author{
Osamu Haba, Yasushi Morimoto, Takahiro UesaKa, \\ Kazuaki YoKota, ${ }^{\dagger}$ and Toyoji KaKUCHI ${ }^{*, \dagger}$ \\ Division of Molecular Chemistry, Graduate School of Engineering, \\ Hokkaido University, Sapporo 060, Japan \\ * Division of Bioscience, Graduate School of Environmental Earth Science, \\ Hokkaido University, Sapporo 060, Japan
}

\begin{abstract}
KEY WORDS Asymmetric Induction / Asymmetric Cyclopolymerization / Optically Active Polymer / Cyclopolymerization / Cyclocopolymerization / Chiral Template / Radical Polymerization / Exciton Chirality Method / Circular Dichroism Spectrum / Absolute Configuration /
\end{abstract}

An optically active vinyl copolymer can be produced through cyclocopolymerization of the divinyl monomer having a chiral template with the vinyl comonomer, followed by removing the template. ${ }^{1}$ This asymmetric cyclocopolymerization method is one specific method to introduce chirality to the polymer main chain. We reported that bis(4-vinylbenzoate)s of chiral diols are suitable monomers for this purpose. ${ }^{2}$ These monomers were cyclocopolymerized with achiral monovinyl compounds, such as styrene, and the resulting copolymers underwent alkali hydrolysis to yield optically active copolymers with main chain chirality. Thus, we have used a wide variety of cyclic chiral diols such as 2,3$O$-isopropylidene-L-threitol ${ }^{2 a}$ (1), 4,6-O-isopropylidene$\alpha, \mathrm{D}-$ gluco- (2), and - $\alpha, \mathrm{D}-\mathrm{mannopyranoside}{ }^{2 \mathrm{~b}}$ (3) to produce an effective asymmetric induction to the main chain and to produce optically active poly(methyl 4vinylbenzoate-co-styrene). The use of acyclic chiral diols such as $(2 S, 4 S)$-2,4-pentanediol ${ }^{2 \mathrm{c}}$ (4) and $(S)$-1,3butanediol $^{2 \mathrm{~d}}(5)$ provides important information about the chiral structural requirement for the template. The most effective template among the five attempts was 4 which is a simple acyclic 1,3-diol and relatively less bulky. The more simple template 5 was less effective than $\mathbf{4}$, but more than the bulky ones such as $\mathbf{3}$. Thus, it is interesting to determine size limitations of the template and what is the smallest but effective chiral template. $(S)$-1,2Propanediol is the smallest member of chiral diols. Here we report the synthesis of the optically active polymer using $(S)$-1,2-propanediyl bis(4-vinylbenzoate) (6) as the monomer having the smallest and simplest chiral template.

Monomer 6, a white powder, was prepared by reacting $(S)$-1,2-propanediol with 4-vinylbenzoyl chloride. Copolymerizations of $\mathbf{6}$ with styrene $(\mathrm{St})$ were carried out using AIBN in toluene at $60^{\circ} \mathrm{C}$. The results are listed in Table I. The polymerization systems were homogeneous and the resulting copolymers (7) were soluble in chloroform and tetrahydrofuran. Number-averaged molecular weights $\left(M_{n}\right)$ of the polymers ranged from 2300 to 17800 . The extent of cyclization $\left(f_{\mathrm{c}}\right)$ of 7 was determined by the area ratio between the vinyl and carbonyl peaks in ${ }^{13} \mathrm{C}$ NMR spectra measured using the inversed-gated decoupling method. When the mole fraction of 6 in the feed $\left(F_{1}\right)$ was 0.05 , the vinyl carbon was not observed in the ${ }^{13} \mathrm{C}$ NMR spectrum of the resulting copolymer. $F_{\mathrm{c}}$ was then decreased to 0.74 along with increase in $F_{1}$. The copolymerization reactivity of 6 was higher than that of $\mathrm{St}$, resulting in the mole fraction of the $\mathbf{6}$ unit in copolymer 7 being higher than that in

Table I. Cyclocopolymerization of $(S)$-1,2-propanediyl bis(4-vinylbenzoate) (6) and styrene (St) and synthesis of poly(methyl 4-vinylbenzoate-co-styrene) (8)

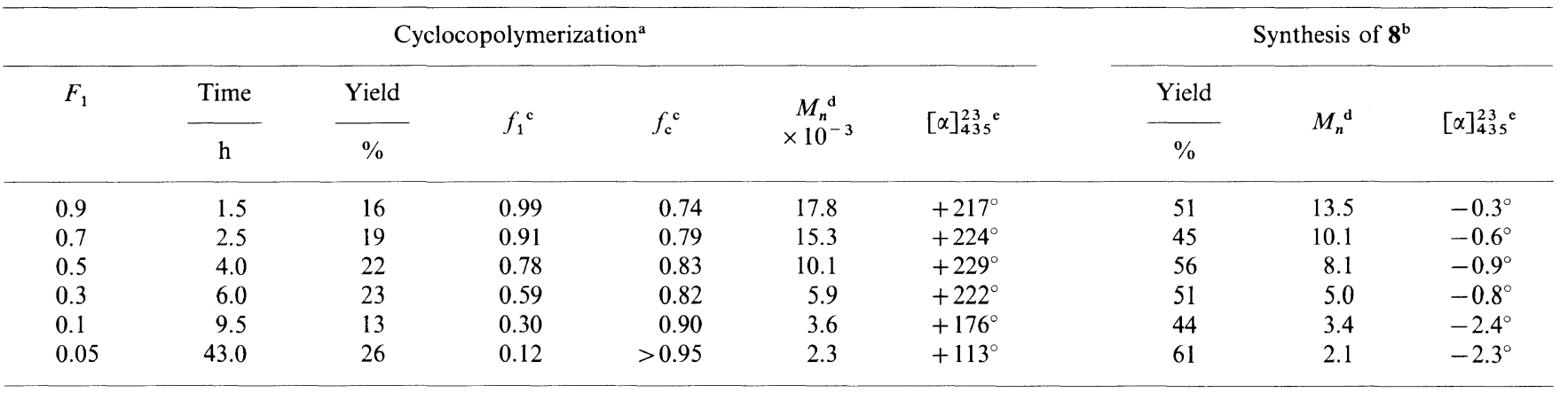

${ }^{\text {a }}$ Solvent, toluene; initiator, AIBN; temp, $60^{\circ} \mathrm{C} ;[6+\mathrm{St}]=0.1 \mathrm{moll}^{-1} ;[\mathrm{AIBN}]=1 \mathrm{gl}^{-1}$. ${ }^{\mathrm{b}}$ Prepared from polymer 7 through hydrolysis using $\mathrm{KOH}$ in aqueous $\mathrm{MeOH}$ for $50 \mathrm{~h}$ under reflux, and then treatment with diazomethane in benzene-ether. ${ }^{\mathrm{c}}$ Determined by ${ }^{13} \mathrm{C}$ NMR spectra. ${ }^{\mathrm{d}}$ Determined by GPC using poly(styrene) standard. ${ }^{\mathrm{e}} \mathrm{Measured} \mathrm{in} \mathrm{CHCl}_{3}$ at $23^{\circ} \mathrm{C}(c=1.0)$.

\footnotetext{
$\dagger$ To whom all correspondence should be addressed.
} 


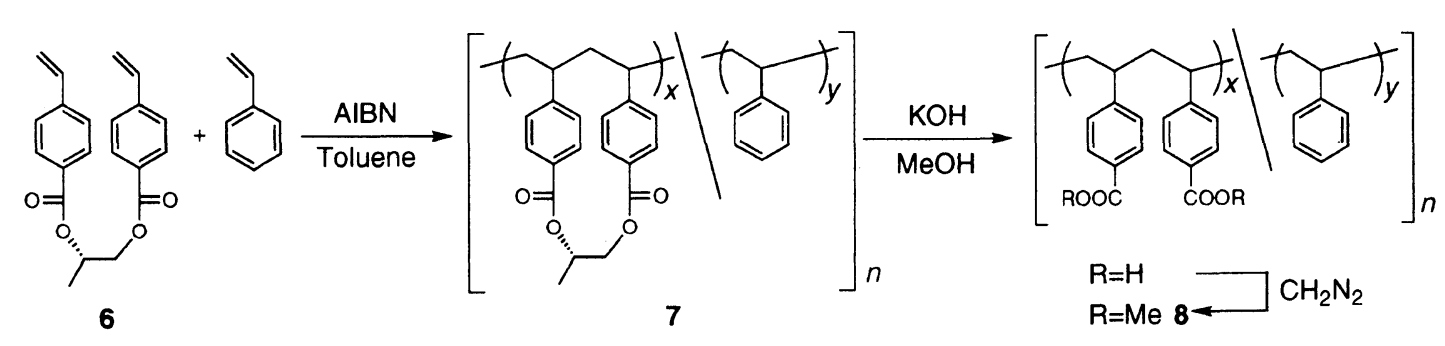

Scheme 1.

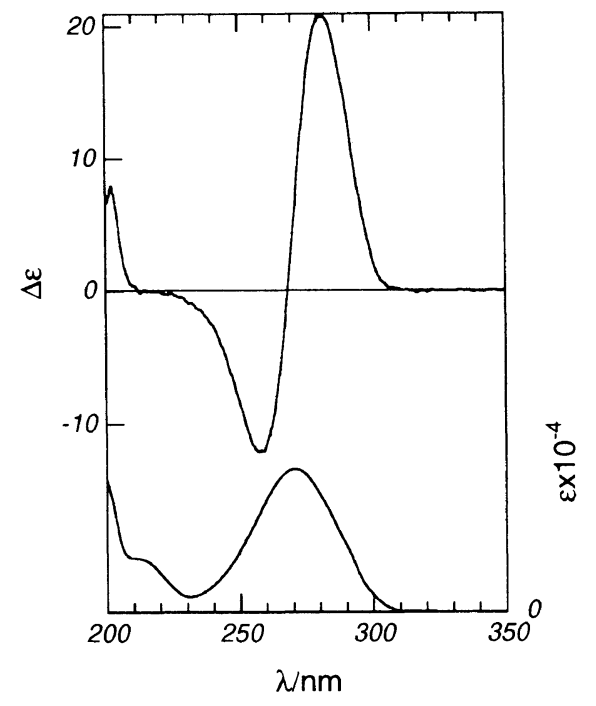

Figure 1. CD and UV spectra of monomer 6, measured in HFP at $21^{\circ} \mathrm{C}$. Sample concentration was $0.211 \mathrm{mg} / 10 \mathrm{ml}\left(6.27 \times 10^{-5} \mathrm{moll}^{-1}\right)$. Path length, $0.5 \mathrm{~cm}$.

the monomer feed. The specific rotation $\left([\alpha]_{435}^{23}, c 1.0\right.$, $\mathrm{CHCl}_{3}$ ) of the cyclopolymers 7 changed from $+113^{\circ}$ to $+229^{\circ}$ along with the copolymer composition.

To study the asymmetric induction in these copolymer systems, it is necessary to quantitatively remove the chiral templates from the polymers. The removal of the chiral template from 7 was performed in aqueous $\mathrm{MeOH}$ using $\mathrm{KOH}$, and the hydrolyzed copolymers were treated with diazomethane to yield poly(methyl 4-vinylbenzoate-costyrene) (8). Table I lists the results of the synthesis of 8. No chiral template remained in polymer $\mathbf{8}$, because the absorption at $4.4-5.6 \mathrm{ppm}$ due to the template disappeared in the ${ }^{1} \mathrm{H}$ NMR spectrum. The obtained copolymer, poly(methyl 4-vinylbenzoate-co-styrene) (8), showed optical activity with specific rotation $\left([\alpha]_{435}^{23}\right.$, $c 1.0, \mathrm{CHCl}_{3}$ ) ranging from $-0.3^{\circ}$ to $-2.4^{\circ}$. The sign of these values is opposite to that for 7 for every composition. The absolute value of rotation increased with decrease of $f_{1}$ and the maximum value was obtained at 0.30 of $f_{1}$. The optical activity of the copolymers is caused by enantiomeric threo diads separeted by comonomeric units. ${ }^{1,2}$ The larger $f_{1}$ reduces the contents of such diads and causes such smaller rotation. These results indicated that a new chirality was induced in the main chain of the monomeric units from 6 by a twist in the $(S)$-1,2-propanediol template.

The CD exciton chirality method ${ }^{3}$ for determining the absolute configurations of bisbenzoate derivatives is quite suitable for analyzing the stereochemistry of monomer 6 and polymer 8 . Figure 1 shows the $C D$ and UV spectra of 6 . The CD spectrum exhibits a positive

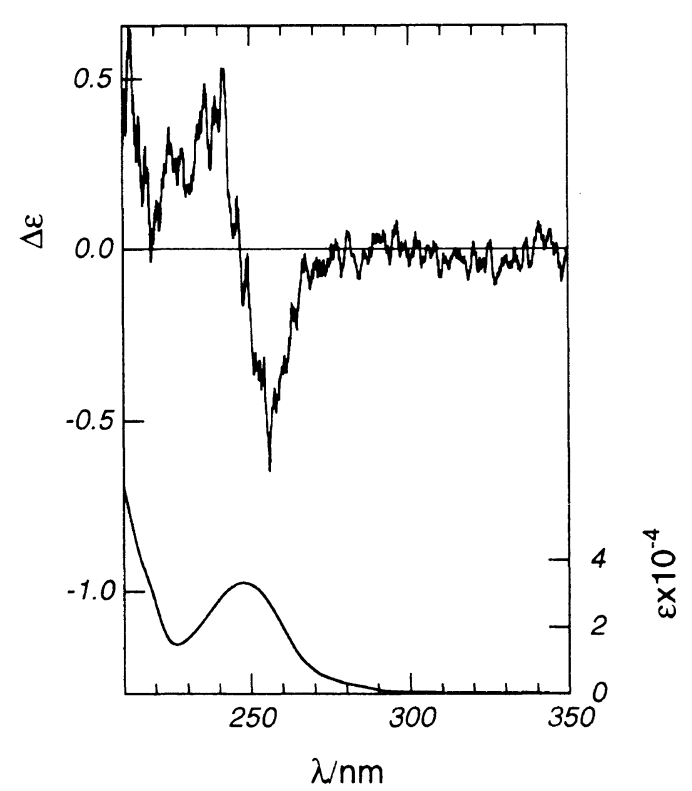

Figure 2. $\mathrm{CD}$ and UV spectra of polymer $8(\mathbf{1} / \mathrm{St}=0.12 / 0.88)$, measured in HFP at $21^{\circ} \mathrm{C}$. Sample concentration was $0.233 \mathrm{mg} / 5 \mathrm{ml}$. $\Delta \varepsilon$ and $\varepsilon$ were based on the concentration of bis(methyl $p$-vinylbenzoate) units $\left(4.24 \times 10^{-5} \mathrm{moll}^{-1}\right)$ calculated using the copolymer composition of 0.12 . Path length, $0.5 \mathrm{~cm}$.

Cotton effect $(\Delta \varepsilon=+20.1)$ at $280.9 \mathrm{~nm}$ and a negative one $(\Delta \varepsilon=-11.7)$ at $258.0 \mathrm{~nm}$ in the region of $\pi \rightarrow \pi^{*}$ transition $\left(\lambda_{\max } 270.8 \mathrm{~nm}\right)$. Monomer 6 thus has positive chirality and its bezoate chromophores, the two 4-vinylbenzoyl groups, twist clockwise. This enantiotopic twist in monomer $\mathbf{6}$ is achieved by one chiral center originating from the $(S)$-1,2-propanediol which is able to act as a chiral template for asymmetric cyclocopolymerization.

The CD spectrum of $\mathbf{8}(\mathbf{6} / \mathrm{St}=0.12 / 0.88)$ showed the split Cotton effect with negative Cotton effect at 256.1 $\mathrm{nm}$ and a positive one at $239.5 \mathrm{~nm}$ (Figure 2). Polymer 8 has negative chirality in which two 4-vinylbenzoyl groups twist counterclockwise resulting in an $(R, R)$ configuration of the vicinal (methyl 4-vinylbenzoate) units in the main chain. Figures 1 and 2 indicate that the chirality of the dibenzoate is positive in monomer 6 but negative in polymer 8 . The $(S)$-1,2-propanediol template having one chiral center transmitted its chirality to the main chain during intramolecular cyclization to form an enantiomeric $R, R$-racemo configuration in the polymer 8.

In summary, optically active poly(methyl 4-vinylbenzoate-co-styrene) was obtained through the radical cyclocopolymerization of $(S)$-1,2-propanediyl bis(4vinylbenzoate) with styrene, followed by removal of the template. This study shows that even the smallest 
template, $(S)$-1,2-propanediol, induces new chirality in the main chain, while its effect is much smaller than 4 and 5. The copolymer 8 with the larger content of $\mathbf{6}$ of over 0.59 , however, showed no significant optical rotation. Thus, $(S)$-1,2-propanediol is a size limited template for asymmetric cyclocopolymerization.

\section{EXPERIMENTAL}

\section{(S)-1,2-Propanediyl Bis(4-vinylbenzoate) (6)}

To a solution of $(S)$-1,2-propanediol (Tokyo Kasei Kogyo Co.) $(5 \mathrm{~g}, 66 \mathrm{mmol})$ in dry pyridine $(300 \mathrm{ml})$ was cooled to $5^{\circ} \mathrm{C}$ in an ice bath. To this solution, a solution of 4-vinylbenzoyl chloride $4(26 \mathrm{~g}, 156 \mathrm{mmol})$ was gradually added so that the temperature of the solution did not rise over $10{ }^{\circ} \mathrm{C}$. The reaction mixture was stirred for $4 \mathrm{~h}$ at $80^{\circ} \mathrm{C}$. The precipitate was filtered off, and the filtrate was successively washed with several portions of aqueous potassium bicarbonate and water, and dried over anhydrous sodium sulfate. After removal of the solvent under reduced pressure, the residue was purified by column chromatography on silica gel with $n$-hexane-ether (4:1) to yield $(S)$-1,2-propanediyl bis(4-vinylbenzoate) (6) $(16.1 \mathrm{~g}, 73 \%) \cdot \mathrm{mp} .49^{\circ} \mathrm{C},[\alpha]_{\mathrm{D}}^{23}=+76.8^{\circ}$, $[\alpha]_{435}^{23}=+199.1^{\circ}\left(c 1.0, \mathrm{CHCl}_{3}\right) \cdot{ }^{1} \mathrm{H}$ NMR $(270 \mathrm{MHz}$, $\left.\mathrm{CDCl}_{3}\right): \delta=7.95-8.02(\mathrm{~m}, 4 \mathrm{H}, \mathrm{Ar}), 7.40-7.45(\mathrm{~m}$, $4 \mathrm{H}, \mathrm{Ar}), 6.74\left(\mathrm{dd}, J_{\text {trans }}=17.8 \mathrm{~Hz}, J_{\text {cis }}=10.9 \mathrm{~Hz}, 1 \mathrm{H}\right.$, $=\mathrm{CH}-), 6.73\left(\mathrm{dd}, J_{\text {trans }}=17.5 \mathrm{~Hz}, J_{\text {cis }}=10.9 \mathrm{~Hz}, 1 \mathrm{H}\right.$, $=\mathrm{CH}-), 5.83\left(\mathrm{~d}, J_{\text {trans }}=17.5 \mathrm{~Hz}, 1 \mathrm{H},=\mathrm{CH}_{2}\right), 5.82(\mathrm{~d}$, $\left.J_{\text {trans }}=17.5 \mathrm{~Hz}, 1 \mathrm{H},=\mathrm{CH}_{2}\right), 5.48-5.59(\mathrm{~m}, 1 \mathrm{H}, \mathrm{CH})$, $5.35\left(\mathrm{~d}, J_{\text {cis }}=10.9 \mathrm{~Hz}, 2 \mathrm{H},=\mathrm{CH}_{2}\right), 4.44-4.55(\mathrm{~m}, 2 \mathrm{H}$, $\left.\mathrm{OCH}_{2}\right), 1.46\left(\mathrm{~d}, J=6.6 \mathrm{~Hz}, 3 \mathrm{H}, \mathrm{CH}_{3}\right) .{ }^{13} \mathrm{C} \quad \mathrm{NMR}$ $\left(67.8 \mathrm{MHz}, \mathrm{CDCl}_{3}\right): \delta=165.8,165.6(\mathrm{C}=\mathrm{O}), 142.0$, $141.9,129.8,129.6,129.2,128.8,126.0$ (arom), 135.8 $(=\mathrm{CH}-), 116.4\left(\mathrm{CH}_{2}=\right), 68.8(\mathrm{CH}), 66.5\left(\mathrm{OCH}_{2}\right), 16.6$ $\left(\mathrm{CH}_{3}\right)$. Anal. Calcd for $\mathrm{C}_{21} \mathrm{H}_{20} \mathrm{O}_{4}$ (336.4): C, 74.98\%; H, 5.99\%. Found: C, $75.08 \%$; H, 5.95\%.

\section{Cyclocopolymerization}

Copolymerizations of $\mathbf{6}$ with styrene $(\mathrm{St})$ were carried out using AIBN in toluene at $60^{\circ} \mathrm{C}$. The detailed procedure is reported in a previous paper. ${ }^{2 a}$

\section{Poly(methyl 4-vinylbenzoate-co-styrene) (8)}

The removal of the chiral template from 7 was carried out using $\mathrm{KOH}$ in aqueous $\mathrm{MeOH}$, and the hydrolyzed copolymers were treated with diazomethane in benzeneether. The detailed procedure is reported in a previous paper. $^{2 a}$

Acknowledgment. This paper was supported in part by a Grant-in-Aid for Scientific Research from the Ministry of Education, Science, and Culture of Japan.

\section{REFERENCES}

1. (a) G. Wulff, K. Zabrocki, and J. Hohn, J. Angew. Chem., Int. Ed. Engl., 17, 535 (1978). (b) G. Wulff and P. K. Dhal, Makromol. Chem., 188, 2847 (1987). (c) G. Wulff, R. Kemmerer, and B. Vogt, J. Am. Chem. Soc., 109, 7449 (1987). (d) G. Wulff and P. K. Dhal, Macromolecules, 21, 571 (1988). (e) G. Wulff and P. K. Dhal, J. Angew. Chem., Int Ed. Engl., 28, 196 (1989). (f) G. Wulff, J. Angew. Chem., Int. Ed. Engl., 28, 21 (1989). (g) G. Wulff and P. K. Dhal, Macromolecules, 23, 100 (1990). (h) G. Wulff and P. K. Dhal, Macromolecules, 23, 4525 (1990). (i) P. K. Dhal, B. Vogt, A. Steigel, and G. Wulff, Macromolecules, 25, 5601 (1992). (j) G. Wulff, H. Schmidt, H. Witt, and R. Zentel, J. Angew. Chem., Int. Ed. Engl., 33, 188 (1994). (k) G. Wulff and S. Krieger, Macromol. Chem. Phys., 195, 3665 (1994).

2. (a) T. Kakuchi, O. Haba, N. Fukui, and K. Yokota, Macromolecules, 28, 5941 (1995). (b) T. Kakuchi, O. Haba, N, Fukui, T. Uesaka, and K. Yokota, submitted to Polymer. (c) O. Haba, Y. Morimoto, T. Uesaka, K. Yokota, and T. Kakuchi, Polymer, in print. (d) O. Haba, Y. Morimoto, T. Uesaka, K. Yokota, and T. Kakuchi, Macromolecules, 28, 6378 (1995).

3. N. Harada and K. Nakanishi, "Circular Dichroic SpectroscopyExciton Coupling in Organic Stereochemistry," Mill Valley, CA and Oxford University Press, Oxford, 1983.

4. A. Hirao, Y. Ishino, and S. Nakahama, Macromolecules, 21, 561, (1988). 